\section{Evaluating the impact of an oncology newsletter as an educational tool}

\author{
By Lynn McAlpine and JoAnn Jones
}

\section{Abstract}

One of the most important responsibilities facing murses today is patient education. It is the focal point of the treatment plan that enables patients to better provide self-care and to adapt to lifestyle changes. Patient education efforts need to be evaluated in order to ensure effectiveness and improve the ways in which education is carried out. This article describes an inexpensive and time-efficient evaluation of a cancer education project, a locally-developed oncology newsletter that demonstrated the value of the written word as an aid in the education of cancer patients, and provided the developers with suggestions for the content of future newsletters. Also described are implications for future evaluations of this type.

\section{Introduction:}

\section{Cancer patient education and evaluation}

There is overwhelming support in the literature for the important role of patient education in health care (Deriderian, 1987; Hagopian, 1991; Rover, 1987). Bartlett (1987), in summarizing the research, states that patient education, although not the whole answer, is an essential element of high quality health care. In cancer education specifically, most professionals are committed to providing it in some way (Deriderian, 1987a; Meade, Diekmann \& Thomhill, 1992).

If the intent of patient education is to effectively meet the needs of patients, then the systematic collection and use of data about patient needs and responses to education are necessary. Not to be ignored are the needs of hospital staff who may also benefit from education about patient treatment.

Evaluations can be conducted with either formative or summative intent (Scriven, 1967). The purpose of formative evaluation is to provide data which can be used to determine how best to improve the object being evaluated at any point during development or implementation. The intention of summative evaluation, on the other hand, is to assess outcomes, the degree to which objectives are accomplished, or the object being evaluated meets the needs of those using it. The following case describes a systematic, simple and cost-effective evaluation strategy which incorporated formative and summative approaches.
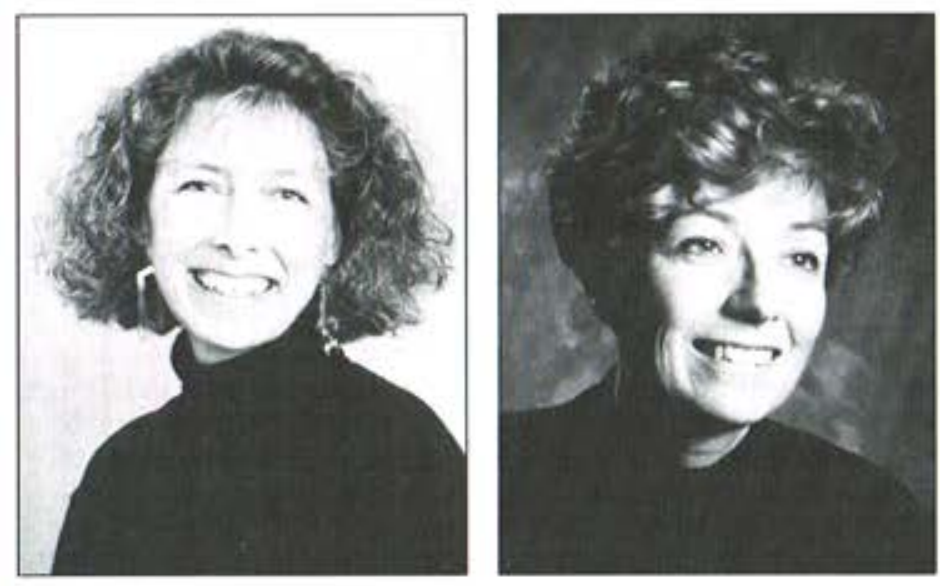

Lynn McAlpine

JoAnn Jones

\section{Goals of the evaluation}

In this instance, the patient education intervention was the development of an oncology newsletter for distribution to patients and hospital staff. Although efforts should be made to evaluate all educational interventions, evaluation was particularly important here as the newsletter was a pilot project. It was important to collect data that could be used to judge the effectiveness of this issue of the newsletter, that is, whether patients perceived their needs were being met with this approach, and to provide the developers with suggestions for the content of futurenewsletters if the project continued.

\section{Context}

The development, distribution and evaluation of the newsletter was undertaken in a university-affiliated, community hospital in a residential area of a large eastern Canadian city. The 272-bed acute care hospital is close to public transportation and draws a mixed cultural group from all over the municipality. The outpatient oncology unit consists of three medical oncologists, one nurse clinician and one part-time nurse consultant.

\section{Impetus for creating the newsletter}

The nurse consultant in the oncology unit became aware that traditional patient education could be more effective if it were supplemented in a written form (Muir-Gray, 1982; Ruzicki, 1989), since the use of written material can reinforce learning and enhance the teaching process (Meade et al, 1992). Anecdotal evidence to support the need for this kind of intervention in the clinical setting included recognition that patients lacked understanding of their disease and its treatment, and patients failed to use information provided by the hospital, eg. not reporting side effects, not knowing how to reach the on-call doctor. These patterns were evident despite staff belief that these items had been taught during patient-staff interactions.

In this particular hospital, as in many hospitals, patient education has largely been provided orally during diagnosis and treatment as the patient interacts with different professional staff. However, evidence suggests that the time of diagnoșis of cancer is not an optimal time for learning to occur. King \& Taylor (1987) and McCorkle \& Ehlke (1993) reported that patients about to undergo chemotherapy neither absorbed nor retained information they needed to cope with treatment despite an indepth consultation with their physician. McCorkle \& Ehlke (1993) reported that, for the patient, the information given during chemotherapy "was like seeing someone move their lips, yet no sounds were heard" (p.13).

Fear, denial and an overload of information are barriers to learning. All of these elements are present during diagnosis and treatment of cancer. The

\section{ÉVALUATION DE L'IMPACT D'UN BULLETIN D'ONCOLOGIE EN TANT QU'OUTIL PÉDAGOGIQUE}

\section{ABRÉGÉ}

De nos jours, l'enseignement aux patients est l'une des plus grandes responsabilités incombant aux infirmières. Cet enseignement est l'élément essentiel du plan de traitement qui permet aux patients d'obtenir de meilleurs résultats au niveau des autosoins et de l'adaptation aux changements de mode de vie. Les efforts réalisés dans ce domaine doivent être analysés afin d'en assurer l'efficacité et d'améliorer les façons dont l'enseignement est prodigué. Cet article décrit la méthode, peu coûteuse et prenant peu de temps, qui a servi à évaluer un projet d'enseignement sur le cancer (un bulletin oncologique développé localement). L'évaluation a démontré que la documentation écrite est un outil de valeur en matière d'éducation des patients souffrant du cancer et a proposé aux auteurs des suggestions sur le contenu des numéros à venir du bulletin. L'article décrit aussi les implications que cela a pour des évaluations futures de ce genre.

Lynn McAlpine, Ed.D is program director, adult education, Faculty of Education, McGill University. JoAnn Jones, RN, MEd is nurse consultant, oncology and palliative care, Queen Elizabeth Hospital, Montreal, Quebec. 
patient has just learned of the threat to his or her health and perhaps existence. As well, many different professionals are providing information on treatment, side effects and possible outcomes within a short period of time. Furthermore, since most of this information exchange is spoken, with little or no visual reinforcement, retention is reduced (Muir-Gray, 1982; Svinicki, 1985). As well, the lack of reinforcing material means the patient cannot revisit the new information at a time more conducive to learning.

Fear and denial, however, represent only two of many modes of coping (Deriderian, 1987a). Deriderian states that other active coping modes are: Information seeking, direct action and turning to others for help and succor. As Deriderian points out, cancer patients may eventually switch to coping strategies such as seeking information about their disease as a way of gaining control. The task for the nurse becomes making the information accessible in this critical time period when the patient evidences readiness to learn(King and Taylor, 1987).

What is needed is an educational tool available at the teachable moment for the patient, an additional form of instruction to supplement or reinforce the traditional oral one so that crucial information is not lost (Harland, Madeley \& Millard, 1992; Meade et al, 1992). In addition, a relatively permanent medium which is easily distributed, portable and accessible for easy reference by both patients and their families is preferred (Hagopian, 1991; Shank et al, 1991). Although there are a number of national publications which meet these requirements, they cannot be written to make familiar the particular people, processes and events that patients will meet in undergoing treatments. Local, personalized information supplements may be more effective because they can be made more specific and contextually rich (Aukerman, 1991; Ruzicki, 1989).

A decision was made to conduct a pilot study of a departmental newsletter for cancer patients. It would provide accessible and practical information to aid in teaching patients and their families; and information to reduce anxiety, to minimize a sense of helplessness and to foster hope. It was not to replace one-on-one counselling and instruction, but was to support these interventions and provide a vehicle for effective follow-up and continuous learning (Meade et al, 1992; Muir-Gray, 1982).

\section{Development of the newsletter}

As Aukerman (1991) and Buchan \& Danku (1989) have provided very clear and concise descriptions of the process of developing and printing a newsletter, the process will not be discussed in detail. A needs assessment was conducted among hospital staff and patients to decide what information to include in the newsletter. The needs assessment focused on the questions most frequently asked by patients and the misunderstandings that most frequently occurred. Hospital staff were asked to contribute articles on these specific topics. Patient volunteers worked with the nursing consultant to edit and produce the newsletter.

Ultimately, 400 newsletters were prepared and printed for distribution. These were sent to the patients in the oncology out-patient department, designated hospital staff and surrounding community agencies, volunteers and interested community members.

The finished product, six pages in length, was printed on good quality paper with a coloured masthead and a number of photographs of contributors and staff. Artwork contributed by a patient portrayed the process of cancer diagnosis from discovery to decision of treatment modality. "Cancer Control" explained the steps taken and identified the various personnel involved in the process. The feature article, written by one of the oncologists, was intended to demystify cancer chemotherapy.

Patients and family caregivers submitted articles on their experiences and their participation as members of the hospital support groups and volunteers. Notices about fund raising activities and requests for assistance were included. Articles on nutrition, commonly misunderstood cancer terms, drug-induced side effects, a description of the resource library and a book review completed the first edition of Cancer Caring and Sharing.

\section{Evaluation of the project}

Evaluation provides an important data base to aid in decision-making. It serves as a management tool that enables one to make well-informed decisions. The data collection and analysis had to provide useful data but be relatively simple to conduct as there was minimal funding for the undertaking. Thus, although it was known that mail-in questionnaires do not have a high return rate, the evaluation took the form of a self-addressed questionnaire inserted in the newsletter, as this method entailed minimal cost (xeroxing of the one-page questionnaire) and a limited amount of time later in collating the results.

The summative concern of the evaluation was the extent to which the newsletter was perceived by patients to be an educational tool. If it was successful, then the formative concern was to leam ways to improve later issues.

\section{The evaluation questionnaire}

The first item requested demographic information so as to compare the impact of the newsletter on different populations. (See Figure One for evaluation form). The second asked readers to rate (on a five-point scale from 'unclear' to 'very clear') the extent to which the goals of the newsletter were clear. Then, as the summative purpose of evaluation was important from a management perspective, the next questions related to different aspects of learning. Learning can focus on attitudinal, cognitive or behavioural changes. In this instance, the cognitive issue was addressed in question \#3 with a request for readers to write examples of what they had learned. Attitude questions followed: \#4 on suitability of format (a five-point scale from 'totally unsuited' to 'very well suited') and \#5 responsiveness to needs (on a five-point scale from 'not at all' to 'very much'). In question \#6, to get formative data to improve future newsletters, respondents were asked to provide a prioritized list of topics they would like to see addressed. Question \#7, a summative one, requested an overall rating of the newsletter (on a five-point scale from 'poor' to 'excellent'). The last item, focusing on formative information, asked those interested in working on future issues to give their names and phone numbers.

The return rate on mailed-out questionnaires tends to be low. In order to improve the chances of return, the evaluation form was printed on bright yellow paper so that it stood out when inserted in the newsletter. As well, an explanation of the importance of completing and retuming the evaluation was given in the last newsletter article "Where do we go from here?". This article explained that the newsletter was a special education project and that response to the newsletter would be a test of its value and would help decide whether future issues would become a reality.

\section{Method of distribution and response rate}

The newsletter with the evaluation was mailed to 248 patients and delivered to 50 staff. (The remainder were kept for later distribution to new patients.) Of the 248 forms mailed to patients, 48 were returned. This low rate of return (19\%) could be attributed to the following factors: a) Patients unwell from either the side effects of their cancer treatments or from the progression of their disease, b) a lack of time for hospital staff to personally solicit written replies. Aside from the written evaluations, many positive oral evaluations were received, but these are not included in the reporting of the results.

Staff return rate was high at $84 \%$. Of the 50 forms given out to staff, 42 were returned. This was likely due to the ease of return through interdepartmental mail or personal delivery, as well asthe fact that a personal note was attached requesting that the written evaluation be completed.

\section{Analysis of data}

The results were collated and analyzed by group: Patient and staff. For the items with rating scales, the number of responses for each rating were summed and percentages tabulated. For the questions requiring written responses, the answers were grouped by theme and percentages tabulated to provide an overall perspective on the respondents' opinions.

\section{Summative results}

\section{Patient response: Cognitive}

Of the 48 patients who responded, $98 \%$ reported that the goals of the newsletter (to provide cancer education and to encourage exchange of experience) were 'very clear'.

In response to the question regarding what was learned from the newsletter, $38 \%$ reported that they primarily leamed what hospital resources were available to them: "Learning how the team pulls together, support groups, roles of individuals and team members". Twenty-seven per cent $(27 \%)$ reported their learning to be the "clear explanation of cancer, its treatment and side effects". Although the expectation had been that this question would access cognitive learning, $30 \%$ of the replies focused on affective outcomes. Nearly a third of patient respondents stated that "caring", 
"not being alone", "there is help", "understanding - hope" was what they had learned.

In the patients' opinion, the most helpful article focused on cancer chemotherapy $(57 \%)$. The next two most helpful articles were cancer control $(18 \%)$ and nutrition $(10 \%)$.

Patient response: Attitudinal

Ninety per cent $(90 \%)$ reported that the newsletter 'very much' met their needs. Ninety-five per cent $(95 \%)$ reported that the format was 'very well suited' for leaming. Thirty-eight per cent $(38 \%)$ of respondents volunteered to assist with future editions.

\section{Staff response: Cognitive}

Ninety-five per cent $(95 \%)$ of the 42 staff members who responded reported the goals of the newsletter (to provide cancer education and to encourage exchange of experience) to be 'very clear'.

Thirty-three per cent $(33 \%)$ reported the most important learning to be the explanations of cancer, treatments and side effects. Twenty-six per cent $(26 \%)$ reported the primary learning was the hospital resources available. Five per cent $(5 \%)$ mentioned nutrition, and two per cent $(2 \%)$ caring. Notably, the first two items are the same as those reported by patients, but in reverse order.

Thirty-eight per cent (38\%) cited the cancer chemotherapy article as the most helpful. The next two choices at $19 \%$ and eight per cent $(8 \%)$, were cancer control and nutrition, respectively. Notably, these were the same articles in the same order as those chosen by patients.

Staff response: Attitudinal

Responses were similar to patient responses. Ninety $(90 \%)$ reported that the newsletter 'very much' met their needs. Ninety-five (95\%) reported that the format was 'very well suited' for learning.

\section{Table One: Patient suggestions for topics for future issues}

Topics for later issues

nutrition

$\%$ of total patient response

treatment of cancer

16

emotional \& physical factors relating to coping

14

cancer types: signs \& symptoms

family involvement

self-examination

self-help groups

\section{Formative results}

\section{Patient response: Cognitive}

The formative question requesting respondents to suggest topics for later issues provided concrete ideas from patients, with information on nutrition the most frequent suggestion (16\%). Table One shows the suggestions with percentage of responses; all suggestions made by more than one individual are included.

\section{Patient response: Attitudinal}

Thirty-eight per cent ( $38 \%$ ) of patients reported that they would like to be involved in the preparation of future newsletters.

Staff response: Cognitive

As with patients, the formative question requesting respondents to suggest topics for later issues resulted in nutrition being the most frequent suggestion, but at a lower percentage (6\%).

Table Two shows the suggestions with percentage of responses; all suggestions made by more than one individual are included. As one would expect, the response rate to this question was lower than that from patients.

\section{Discussion}

\section{Summative evaluation}

It is evident based on the results from those who responded that the outcomes were positive. The data provided confirmation that patients perceived that the information in the newsletter could be assimilated at a time that was personally appropriate; these data also suggested ways in which future newsletters could continue to meet needs. However, since the patient responses represent only $19 \%$ of the population, caution must be used in interpreting these results. For instance, there may be topics of interest to other patients than those cited (although the fact that there was considerable overlap with staff provides some measure of confidence). As well, the

\section{Table Two: Staff suggestions for topics for future issues}

Topics for later issues

$\%$ of total staff responses

nutrition 6

emotional \& physical factors related to coping

5

family involvement

self-help groups

treatment of cancer

cancer types: signs \& symptoms
4

3

\section{Figure One: Newsletter evaluation}

1. Background information: Which describes you best? (Please check appropriate space)

$\square$ Patient $\square$ Family member (please specify) Friend Age

$\square$ Professional $\square$ Volunteer $\square$ Other (please specify) Sex

2. The purpose of this newsletter is to provide cancer education and to encourage exchange of experiences.

How clear were these goals to you? unclear/ / very clear

3.a) What did you learn from this newsletter that was helpful?

b) Which article was most helpful for you? (Identify by title)

Comments

4. How well was the format, presentation, etc. suited to learning?

totally unsuited / / very well suited

5. How well did this newsletter respond to your needs and/or interest? not at all / / very much

6. Please list any issues or topics which you would like to see followed up or dealt with in more depth, and rank them in order of priority of importance to you.

7. How would you rate this newsletter? poor/ lexcellent

8. If you are interested in participating or contributing to a second edition, please leave your name and phone number: Name: Phone No.

Thank you for your cooperation - your response is needed.

It will determine if future editions will be published, and provide the basis to obtain funding for this project. 
newsletter may be useful for only a limited number of patients, that is, this format may not be accessible at teachable moments for some patients because they have forgotten they have it or they have trouble reading it. However, no single instructional strategy can be effective with every learner. Given the many positive oral comments made by patients who did not complete written evaluations, we can safely assume that the $20 \%$ in this sample are representative of a larger group of patients. In other words, in this setting the newsletter was useful for a minimum one in five patients.

It is apparent in assessing staff responses that the newsletter was an educational tool for them as well. One cannot assume that those working in specialized fields always have a comprehensive understanding of all the content that patients are expected at some level to understand. Locally developed newsletters may also target staff as secondary populations of learners.

Although there was a mixture of articles, some personal stories, some local hospital news and some educational pieces, the most helpful items cited by patients were all in the last category. This supports the notion that, although patients have received information about their treatment in conversation with hospital personnel, written reinforcement is helpful. Staff also reported these articles as the most helpful.

\section{Formative evaluation}

Useful formative findings emerged. One was a clear delineation of future learning needs: Information on nutrition, treatment, types of cancer and prevention. This was useful information for the nurse consultant, and likely had a positive value for the patients themselves. Patients are the best source for identifying their educational needs (Knowles, 1985) and they benefit from the opportunity to express these needs (Deriderian, 1987b).

Another finding concerned patient interest in working on the preparation of the newsletter. Initially, the nurse consultant had involved patients in preparing the newsletter, but by the end of the project had largely abandoned the activity because of difficulties relating to the progression of patients' diseases and side effects of treatment. However, the positive response on the evaluation form regarding patient involvement led her to reconsider this position and decide to continue to involve patients in future issues, whatever the difficulties.

\section{Unexpected outcomes}

The collection of evaluation data often provides unexpected but useful insights. Based on open-ended responses to the cognitive items, patients perceived the newsletter as a manifestation of caring on the part of the oncology department. This is an asset for the hospital in terms of its ongoing relationship with patients as well as its public relations with the community. Another unexpected outcome was the learning cited by staff.

\section{Long-term impact of the evaluation}

After initial distribution, requests were received for additional copies, so funding was made available to print another 400 newsletters. The in-service coordinator began using the newsletter for new staff orientation. As well, a reprint of the articles cited as the most helpful, "Cancer chemotherapy" and "Cancer control", was prepared to be given to all new patients on their initial visit to the oncology clinic.

Perhaps most important to report is that the results were instrumental in obtaining financial support from a pharmaceutical company and from patients for a quarterly newsletter. Without the evaluation data, it would have been difficult to convince funding sources that the project was of value.

\section{Implications for future evaluations}

This evaluation was the first attempt in this setting to systematically collect information from patients on the impact of patient education. As with all such attempts, lessons for future evaluations were learned. One is the necessity of improving the return rate. Possibilities are: Using a systematic collection of oral feedback from patients through phone calls, collecting feedback during hospital visits using an oral protocol, or tracking all unsolicited comments and using them to initiate a short oral protocol. Another lesson is to think about forms of impact beyond patients' perceived learning and satisfaction which were the areas the evaluation form focused on. The form didn't, for instance, provide information about transfer of learning. One possibility here would be to track if the information in the newsletter leads to changes in patient behaviour, eg. does additional written reinforcement about the correct use of anti-emetics or pain medication affect patient compliance? Another area to be explored would be the impact of the newsletter on the institution over time, eg. do staff find they are answering more appropriate questions as a result of the information given in the newsletter?

\section{Conclusion}

If formal patient education is to become more effective, we need to have answers to many questions about how best to facilitate learning when people are sick and under stress. Small practice-based inquiries that are relatively cost- and time-effective can provide useful data for management decisions, and add to our understanding of how to improve cancer patient education programs. As well, inquiries of this kind can bring to light unexpected outcomes, outcomes that might be overlooked without systematic data collection and analysis.

The challenge to oncology nurses is to provide effective education by responding sensitively to patients' perceived learning needs. This entails actively seeking information in order to guide the development of learning materials. Evaluations of the kind described can provide such guidance.

\section{References}

1. Aukerman, G.F. (1991). Developing a patient education newsletter. The Journal of Family Practice, 33(3), 304-305.

2. Bartlett, E. (1987). The educational model of health care. The Canadian Nurse, 3.

3. Buchan, D. \& Danku, L. (1989, Nov.). Communicating excellence. The Canadian Nurse, Oct., 22.

4. Deriderian, A. (1987a). Informational needs of recently diagnosed cancer patients: A theoretical framework (Part I). Cancer Nursing, 10(2), 107-115.

5. Deriderian, A. (1987b). Informational needs of recently diagnosed cancer patients: A theoretical framework (Part II). Cancer Nursing, 10(3), 156-163.

6. Hagopian, G.A. (1991). The effects of a weekly radiation therapy newsletter on patients. Oncology Nursing Forum, 18(7), 1199-1203. 7. Harland, C.C., Madeley, R.J. \& Millard, L.G. (1992). Information leaflets in the dermatology out-patient waiting area. British Journal of Dermatology, 127(5), 492-496.

8. James, M. \& Lantz, H. (1987). Building the framework to support high quality projects. The Canadian Nurse, Nov, 35 .

9. King, T. \& Taylor, C. (1987, Oct.). The outpatient way. The Canadian Nurse, 23-25.

10. Knowles, M.S. (1985). Applications in continuing education for the health professions. Mobius, 5(2), 80-100.

11. McCorkle, R. \& Ehlke, G. (1993). Both sides of the fence. International Cancer Nursing News, 5(1), 12-14.

12. Meade, C.D., Diekmann, J. \& Thornhill, D.G. (1992). Readability of American Cancer Society patient education literature. Oncology Nursing Forum, 19(1), 51-55.

13. Muir-Gray, J.A. (1982). Preparing a leaflet for patient education. British Medical Journal, 284, 1171-1172.

14. Rover, R. (1987). Changing our focus. The Canadian Nurse, Oct, $12-15$.

15. Ruzicki, D.A. (1989). Realistically meeting the educational needs of acute and short-term hospitalized patients. The Nursing Clinics of North America, 24(3), 629-637.

16. Scriven, M. (1967). The methodology of evaluation. Curriculum Evaluation, Monograph Series on Evaluation No. 1, Stake, R.E. (ed.). Chicago, III.: Rand McNally.

17. Svinicki, M. (1985). "It ain't necessarily so": Uncovering some assumptions about learners and lectures. To Improve the Academy, 134-144. 